\title{
Motion of an active particle with dynamical disorder
}

\author{
Koushik Goswami* and Rajarshi Chakrabarti** \\ Department of Chemistry, \\ Indian Institute of Technology Bombay, India \\ *E-mail: goswamikoushik10@gmail.com \\ **E-mail: rajarshi@chem.iitb.ac.in and
}

Published in Soft Matter, 2022, 10.1039/D1SM01816G

\begin{abstract}
We propose a model for the motion of a single active particle in a heterogeneous environment where the heterogeneity may arise due to the crowding, conformational fluctuations and/or slow rearrangement of the surroundings. Describing the active particle in terms of the Ornstein-Uhlenbeck process (OUP) and incorporating the heterogeneity in the thermal bath using the two separate models, namely "diffusing diffusivity" and "switching diffusion", we explore the essential dynamical properties of the particle for its one-dimensional motion. In addition, we show how the dynamical behavior is controlled by dynamical variables associated with the active noise such as strength and persistence time. Our model is relevant in the context of single particle dynamics in crowded environment, driven by activity.
\end{abstract}

\section{INTRODUCTION}

Recent advances in the single-particle tracking technique have promoted the studies on the motion of single particles which are of great importance as they provide deep insights about the nonequilibrium physics, in addition to their immense potential applicability in the field of biophysics [1, 2]. In biological systems, from cargo transport by a motor protein to the motion of a microorganism such as bacteria, a particle moves on its own by harnessing energy from an active process like ATP hydrolysis. Such system is termed as active and it operates out of equilibrium [3, 4]. One of the most common ways of modelling an active particle is by describing its propulsion velocity as the Ornstein-Uhlenbeck process (OUP), and it is termed as the "active Ornstein-Uhlenbeck particle" (AOUP) model [5-8]. In this model, the velocities at different times are exponentially correlated [6, 9]. Therefore, the motion has persistence. By construction, in the AOUP framework, the particle has a Gaussian distribution in space, and it ensures enhanced diffusion in the long-time limit. 
It is worth mentioning here that the OUP model can also be adapted to describe the dynamics of passive particles immersed in a bath containing active particles, e.g., see Refs. [10-14]. The above description is well suited for the diffusion in an environment where the diffusivity of the particle remains unchanged over the time, or in other words, the particle moves in a homogeneous environment.

Over the past decade, experiments on the dynamics of passive tracers in a crowded environment as well as computer simulations have been performed by several research groups [15-21]. The examples include diffusion of lipid vesicles in a solution of entangled filaments [16], tracer diffusion in an environment consisting of large particles or polymer melts or suspension of polymer chains [17, 22, 23], mobile biomolecules on a surface [19, 20], in-plane diffusion of drug molecules in between silica slabs [24], to name but a few. The most notable observation of these studies is the fact that particle has non-Gaussian position distribution. In many cases, the distribution has an exponential tail at intermediate times, which vanishes in the long-time limit, exhibiting a Gaussian behavior, as predicted by the central limit theorem [23, 25]. Interestingly, some studies have shown that though the distribution is non-Gaussian, the mean square displacement (MSD) all times grows linearly in time, or in other words, the dynamics is Fickian [15-17, 21]. Such observations are not consistent with the description of the normal diffusion, and thus the processes associated with similar features are, in general, termed as "anomalous, yet Brownian diffusion" (AYBD) [26, 27]. A plausible explanation for AYBD comes from a theoretical formalism where the tagged particle possesses multiple diffusion coefficients, i.e., the diffusivity is a stochastic variable and it follows some distribution [26]. The idea of introducing fluctuations into intensive variables such as energy dissipation can be traced back to the work on atmospheric turbulence by Obukhov and Kolmogorov [28-31]. Later similar idea was invoked by Beck and Cohen to show how an effective statistical description can be obtained after averaging over the fluctuations of intensive variables such as temperature (or thermal diffusivity), and they called it "superstatistics" [32]. However, it was Robert Zwanzig who, in a different context, introduced essentially the same concept in a more general way while describing the rate process with a rate constant that fluctuates in time due to fluctuations of the barrier height [33]. It can take discrete values or it can be a continuous function. Zwanzig called this "dynamical disorder" [23, 34, 35]. In the context of diffusing particles, a similar formalism named "diffusing diffusivity" (DD) model was put forward by Chubynsky and Slater [26], and it was further extended by Sebastian's group and Metzler's group independently [36-41]. The model of Chubynsky and Slater is a case of static 
(or quenched) disorder as the diffusivity does not fluctuate with time as it has a stationary distribution. But for the models developed by Sebastian and Metzler, the diffusivity of a particle in a crowded, heterogeneous surroundings is a time-dependent random variable which is considered as the square of a random process such as OU process, since the diffusivity can take only positive values. But when the motion is sampled over all trajectories, it results diffusive motion with the ensemble-averaged diffusivity. In other words, the mean square displacement is proportional to the time elapsed, i.e., $\left\langle x^{2}(t)\right\rangle=K t$, and the coefficient of proportionality $(K)$ is fully time-independent quantity which can be expressed in terms of the equilibrium diffusivity. This model is applied to describe the motion in a heterogeneous environment rearranging with a timescale comparable to the one corresponding to the diffusing particle. It should be noted here that in the quencheddisordered media the DD approach can also be applied but only in the limit of rapidly changing disorder [42-44]. For different random-diffusivity models, readers are referred to Refs. [45-47]. See also Ref. [48] for the derivation of AYBD using the large deviations approach. There are situations where the particle can have few conformations specified by their diffusivities, or there are spatial heterogeneity or specific kinds of interaction between the particle and its surroundings which leads to a motion associated with discrete diffusivities [49-52]. For instance, a freely diffusing particle intermittently trapped due to presence of random binding zones or traps exhibits a non-Gaussian spatial distribution [53]. Another example includes conformational fluctuations of a protein called transcription factor which switches between two conformations while searching for a promoter site to initiate the transcription; in one conformation it moves fast on the DNA track, but in another conformation it undergoes a slow motion due to strong interaction with the track [50]. The above dynamics is usually described by the two-state model or the switching diffusion [35, 54]. Apart from the Brownian dynamics, applications of the switching model in describing several biochemical processes such as cellular signalling, chemotaxis, synaptic dynamics, growth of cell population, pattern formation are noteworthy in relation to the present topic [55-59]. In addition to the AYB diffusion of Brownian particles, stochasticity in diffusivity naturally arises in the dynamics of macromolecules such as conformational fluctuations of proteins |60, 61] and the motion of the center of mass of (de)polymerising or shape-shifting molecules [61, 62]. The nonGaussian behavior can also be observed for sub-diffusing particles moving in gels or viscoelastic media such as cytoplasmic environment due to heterogeneity [63-66]. See the theoretical work of Ref. [67] where unexpected non-Gaussianity occurs for Lennard-Jones mixtures as a result of intermittent hopping motion. 
In all the examples discussed above on the heterogeneous diffusion, the particle's motion is mainly influenced by the thermal fluctuations, or more specifically, no intrinsic energy source or no external stochastic driving is considered, and therefore, the dynamics is in equilibrium. However, as mentioned earlier, the diffusive motion coupled to an active process is very common in biological systems, though its theoretical study in heterogeneous environment is largely lacking. Inclusion of heterogeneity is important to study the activity-driven systems such as self-propelled particles in the presence of obstacles [68], active soft colloids with switching active interactions [69], runand-tumble disks displaying avalanche dynamics [70], transport of active tracers in a polymer grafted channel [71], etc. For a detailed account on this topic, the reader is referred to Ref. [72]. Along these lines, there have been theoretical investigations of the impact of heterogeneity on the transport properties of active particles [73, 74]. These studies have mostly considered the active Brownian particle (ABP) model to describe the dynamics, and the fluctuations are incorporated either in self-propulsion velocity or both in thermal and active forces. In another study [69], Bley et. al. have investigated the dynamical properties of a suspension of active colloids where each particle switches randomly between two states differing in their size as well as mobility. It has been shown there that this switching induces non-Gaussian behavior at intermediate times.

Drawing motivations from these experimental and simulation based investigations, here we propose an analytically solvable model to capture the dynamics of a simple one-dimensional active particle with dynamical disorder. We consider two cases, namely the switching diffusion where the particle switches between two conformations with two different thermal diffusivities, and the diffusing diffusivity model in which the particle takes instantaneous thermal diffusivity which changes randomly over time due to the rearrangement of the environment. In our model, the activity does not get affected by the heterogeneity, and it is modeled as Ornstein-Uhlenbeck process. These models along with the dynamics of the particle is discussed in Sec. III In Sec. III we showcase the main results. A summary is given in Sec. IV

\section{DYNAMICS}

Here we consider an active particle moving in a heterogeneous media as pictorially depicted in Fig. 1. For simplicity, the motion is restricted to one dimension although it can be in principle extended to higher dimensions. In the following section, more details about the dynamics is provided. 


\section{A. Model of active noise}

Apart from thermal kicks experienced by the particle, it is also subjected to an extra noise termed as the active noise, and as a result, it executes directed motion. Therefore, the dynamics of the particle is governed by the following stochastic equation:

$$
\dot{x}(t)=\eta_{T}(t)+v_{A}(t),
$$

where $\eta_{T}(t)$ is the thermal noise modeled as a Gaussian white noise with zero mean. Thus, $\left\langle\eta_{T}(t)\right\rangle=0$, and the autocorrelation function is

$$
\left\langle\eta_{T}(t) \eta_{T}\left(t^{\prime}\right)\right\rangle=2 D_{T}(t) \delta\left(t-t^{\prime}\right) .
$$

Here it is assumed that either the environment is crowded and stochastically rearranging, that is to say, the particle explores the heterogeneous environment, or the particle itself alters between many states, which results only the fluctuations in the thermal diffusivity $D_{T}$. Following Zwanzig, it is referred here as the dynamically disorder system. To account for the disorder, two different models are discussed in Sec. IIIB.

On the other hand, $v_{A}(t)$ corresponds to the self-propulsion velocity of the particle, and it can be conceived as the active noise which drives the particle out of equilibrium. As a standard model, the noise taken here is a Gaussian colored noise, which follows the OUP of the form

$$
\dot{v}_{A}(t)=-\frac{1}{\tau_{A}} v_{A}(t)+\frac{1}{\tau_{A}} \eta_{A}(t),
$$

with $\eta_{A}(t)$ being the Gaussian white noise having correlation $\left\langle\eta_{A}(t) \eta_{A}\left(t^{\prime}\right)\right\rangle=2 D_{A} \delta\left(t-t^{\prime}\right)$. So the auto-correlation function of $v_{A}(t)$ can be expressed as

$$
\left\langle v_{A}(t) v_{A}\left(t^{\prime}\right)\right\rangle=\frac{D_{A}}{\tau_{A}} e^{-\frac{\left|t-t^{\prime}\right|}{\tau_{A}}},
$$

where $D_{A}$ denotes the active diffusivity and $\tau_{A}$ is the persistence time. Other than the strength $D_{A}$, the characteristic timescale $\tau_{A}$ characterizes the active noise, and higher value of $\tau_{A}$ means a long-lived directed motion. In the limit $\tau_{A} \rightarrow 0$, the noise becomes delta-correlated and behaves like the thermal noise [75].

\section{B. Model of thermal diffusivity}

As discussed earlier, the thermal diffusivity of the particle fluctuates in time due to disorder. In the following sections, two models are considered to describe the time-dependent diffusivity $D_{T}(t)$. 


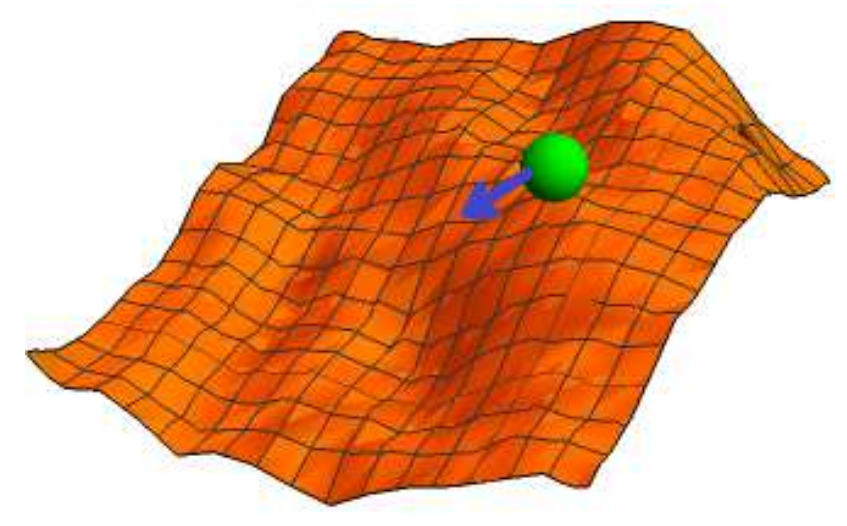

Figure 1. A typical illustration of a diffusing active particle (green ball) in a disordered or heterogeneous medium. The blue arrow represents the instantaneous direction of the self-propulsion velocity $\left(v_{A}\right)$ of the particle.

\section{Switching diffusion}

In the switching diffusion model, the particle switches between two states with two different thermal diffusivities $D_{1}$ and $D_{2}$. Let us call the states as state 1 and state 2 , respectively. The transition happens from the state 2 to the state 1 with a rate $r_{21}$, and vice versa with a rate $r_{12}$. This is a simple version of the "Markov additive" model commonly used to capture the protein movement on a DNA track where it encounters different states of chromatin with variable affinities, and as a result, its motion accounts for the heterogeneous environment [76]. Here we assume that the particle has two discrete states and it starts its journey either from the state 1 or state 2 with their respective steady-state probabilities which are given by

$$
p_{1, s}=\frac{r_{21}}{r_{12}+r_{21}}, \quad p_{2, s}=\frac{r_{12}}{r_{12}+r_{21}}
$$

where $p_{i, s}$ being the steady-state probability at state $i, i=1,2$. For such a case, the characteristic functional can be expressed as [77]

$$
\left\langle e^{-p^{2} \int_{0}^{t} d t^{\prime} D\left(t^{\prime}\right)}\right\rangle_{D\left(t^{\prime}\right)}=\frac{1}{2}\left[1-\frac{\phi_{3}}{\phi_{2}}\right] e^{-\left(\phi_{1}+\phi_{2}\right) t}+\frac{1}{2}\left[1+\frac{\phi_{3}}{\phi_{2}}\right] e^{-\left(\phi_{1}-\phi_{2}\right) t},
$$

where $\phi_{1}=\frac{p^{2}}{2}\left(D_{1}+D_{2}\right)+\frac{1}{2}\left(r_{12}+r_{21}\right), \phi_{2}=\frac{1}{2} \sqrt{\left[\left(D_{2}-D_{1}\right) p^{2}+\left(r_{21}-r_{12}\right)\right]^{2}+4 r_{12} r_{21}}$, and $\phi_{3}=$ $\frac{1}{2}\left(r_{12}+r_{21}\right)-\frac{p^{2}}{2} \frac{r_{12}-r_{21}}{r_{12}+r_{21}}\left(D_{2}-D_{1}\right)$. The equilibrium diffusivity is given by $D_{e q}=\langle D(t)\rangle=p_{2, s} D_{2}+$ $p_{1, s} D_{1}$. Here, $D_{2} \geq D_{1} \geq 0$. 


\section{Diffusing diffusivity model}

In the diffusing diffusivity model, $D_{T}(t)$ is considered as the position of $n$-dimensional harmonic oscillator, i.e., $D_{T}(t)=\sum_{i=1}^{n} \xi_{i}^{2}(t)$, where the evolution of $\xi_{i}(t)$ is governed by the OrnsteinUhlenbeck process (OUP), $\dot{\xi}_{i}=-\omega \xi_{i}(t)+\eta_{i}(t)$. Here $\eta_{i}(t)$ is the white Gaussian noise with correlation $\left\langle\eta_{i}(t) \eta_{j}\left(t^{\prime}\right)\right\rangle=D_{e q} \omega \delta_{i j} \delta\left(t-t^{\prime}\right)$, and $D_{e q}$ is the effective or equilibrium diffusivity of the particle in the medium. Here, we consider a two-dimensional model, i.e., $D_{T}(t)=\sum_{i=1}^{2} \xi_{i}^{2}(t)$. The characteristic functional for such model can be found easily by the path integral technique, and it reads [36]

$$
\left\langle e^{-p^{2} \int_{0}^{t} d t_{1} D\left(t_{1}\right)}\right\rangle_{D\left(t_{1}\right)}=\frac{4 \omega \beta e^{-(\beta-\omega) t}}{(\beta+\omega)^{2}-(\beta-\omega)^{2} e^{-2 \beta t}},
$$

with $\beta=\sqrt{\omega^{2}+2 \omega D_{e q} p^{2}}$. The relaxation timescale for the environment rearrangement is roughly related to the inverse of $\omega$, which implies that for higher values of $\omega$, the environment relaxes faster and as a result, the particle visits all possible configurations of the environment comparatively at a shorter timescale.

\section{RESULTS}

Here our aim is to find the dynamical properties of the particle. To do so, we first outline the technique to obtain the probability distribution function (PDF) in space. Using Eq. (1D), one can write

$$
x(t)=x_{0}+\int_{0}^{t} d t_{1} \eta_{T}\left(t_{1}\right)+\int_{0}^{t} d t_{1} v_{A}\left(t_{1}\right),
$$

with $x_{0}$ being the initial position of the particle. Now the probability distribution function (PDF) of finding the particle at position $x$ at time $t$ considering $x(0)=x_{0}$, can be expressed as

$$
P\left(x, t \mid x_{0}, 0\right)=\langle\delta(x-x(t))\rangle,
$$

where $\langle\cdots\rangle$ represents the ensemble average over all trajectories of $x(t)$. Writing the definition of delta functional explicitly in the above and with the aid of Eq. (8), the PDF can be rewritten as

$$
\begin{aligned}
& P\left(x, t \mid x_{0}, 0\right) \\
& =\frac{1}{2 \pi} \int_{-\infty}^{\infty} d p e^{-i p x}\left\langle e^{i p x(t)}\right\rangle \\
& =\frac{1}{2 \pi} \int_{-\infty}^{\infty} d p e^{-i p\left(x-x_{0}\right)}\left\langle e^{i p \int_{0}^{t} d t^{\prime} \eta_{T}\left(t^{\prime}\right)}\right\rangle_{\eta_{T}\left(t^{\prime}\right)}\left\langle e^{i p \int_{0}^{t} d t^{\prime} v_{A}\left(t^{\prime}\right)}\right\rangle_{v_{A}\left(t^{\prime}\right)} .
\end{aligned}
$$


Now the averages are taken over the noises. As the evolution of the two noises are decoupled to each other, the ensemble averages now can be computed separately as shown in the above equation. It is useful to note here that the fourth-order autocorrelation function of any Gaussian noise $\sigma_{G}$ can be expressed as [78]

$$
\begin{aligned}
& \left\langle\sigma_{G}\left(t_{1}\right) \sigma_{G}\left(t_{2}\right) \sigma_{G}\left(t_{3}\right) \sigma_{G}\left(t_{4}\right)\right\rangle \\
& =\left\langle\sigma_{G}\left(t_{1}\right) \sigma_{G}\left(t_{2}\right)\right\rangle\left\langle\sigma_{G}\left(t_{3}\right) \sigma_{G}\left(t_{4}\right)\right\rangle+\left\langle\sigma_{G}\left(t_{1}\right) \sigma_{G}\left(t_{3}\right)\right\rangle\left\langle\sigma_{G}\left(t_{2}\right) \sigma_{G}\left(t_{4}\right)\right\rangle \\
& +\left\langle\sigma_{G}\left(t_{1}\right) \sigma_{G}\left(t_{4}\right)\right\rangle\left\langle\sigma_{G}\left(t_{3}\right) \sigma_{G}\left(t_{2}\right)\right\rangle
\end{aligned}
$$

and the characteristic functional of the noise $\sigma_{G}$ can be evaluated using the relation [79]

$$
\left\langle e^{i \int_{0}^{t} d t^{\prime} p\left(t^{\prime}\right) \sigma_{G}\left(t^{\prime}\right)}\right\rangle_{\sigma_{G}\left(t^{\prime}\right)}=e^{-\frac{1}{2} \int_{0}^{t} d t_{1} \int_{0}^{t} d t_{2} p\left(t_{1}\right)\left\langle\sigma_{G}\left(t_{1}\right) \sigma_{G}\left(t_{2}\right)\right\rangle p\left(t_{2}\right)}
$$

By the virtue of the above relation and Eq. (4), the average over the active noise can be easily obtained, and it reads

$$
\left\langle e^{i p \int_{0}^{t} d t^{\prime} v_{A}\left(t^{\prime}\right)}\right\rangle_{v_{A}\left(t^{\prime}\right)}=e^{-\frac{p^{2}}{2} \int_{0}^{t} d t_{1} \int_{0}^{t} d t_{2}\left\langle v_{A}\left(t_{1}\right) v_{A}\left(t_{2}\right)\right\rangle}=e^{-D_{A} p^{2}\left(t+\tau_{A}\left(e^{-\frac{t}{\tau_{A}}}-1\right)\right)} .
$$

Similarly for the thermal noise, the average can be calculated using Eq. (2) as

$$
\left\langle\left\langle e^{i p \int_{0}^{t} d t^{\prime} \eta_{T}\left(t^{\prime}\right)}\right\rangle\right\rangle_{\eta_{T}, D_{T}}=\left\langle e^{-p^{2} \int_{0}^{t} d t^{\prime} D_{T}\left(t^{\prime}\right)}\right\rangle_{D_{T}\left(t^{\prime}\right)}
$$

Since the the diffusivity is also a stochastic variable, one also needs to do the averaging over all realizations of $D_{T}(t)$, as shown in the above equation. Now we can take different models of $D_{T}(t)$ as discussed in Sec. ПB to do the further analysis.

To get a preliminary idea about the dynamics, we find the mean square displacement (MSD). For both the models of $D_{T}(t)$, the MSD can be expressed using Eq. (8), and it yields (see Eq. (A1))

$$
\begin{aligned}
& \left\langle\left(x(t)-x_{0}\right)^{2}\right\rangle \\
& =\int_{0}^{t} \int_{0}^{t} d t_{1} d t_{2}\left\langle\eta_{T}\left(t_{1}\right) \eta_{T}\left(t_{2}\right)\right\rangle+\int_{0}^{t} \int_{0}^{t} d t_{1} d t_{2}\left\langle v_{A}\left(t_{1}\right) v_{A}\left(t_{2}\right)\right\rangle \\
& =2 D_{e q} t+2 D_{A}\left(t+\tau_{A}\left(e^{-\frac{t}{\tau_{A}}}-1\right)\right) .
\end{aligned}
$$

Notice that the first term of the right-hand side (RHS) is a linear function of time and is solely coming from the thermal contribution, which we denote here as $\left\langle\left(x(t)-x_{0}\right)^{2}\right\rangle_{T}$. Now we can 


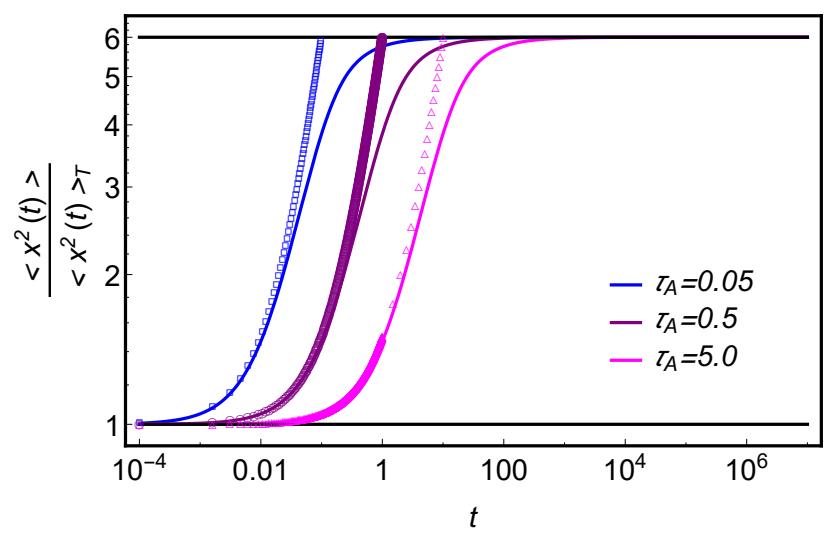

Figure 2. Log-Log plot of the mean square displacement (MSD) [Eq. (15)] scaled by the MSD in a purely thermal bath as a function of time $t$ for different values of $\tau_{A}$. The curves with symbols are the approximate results for the ballistic regime in the limit $t \ll \tau_{A}$ [see Eq. [16)], and two black lines correspond to the very short-time (lower line) and long-time (upper line) diffusive limits. The other parameters used in the plots are $D_{e q}=5.0, D_{A}=25, x_{0}=0$.

distinguish different time limits for which the MSD can be approximated as follows:

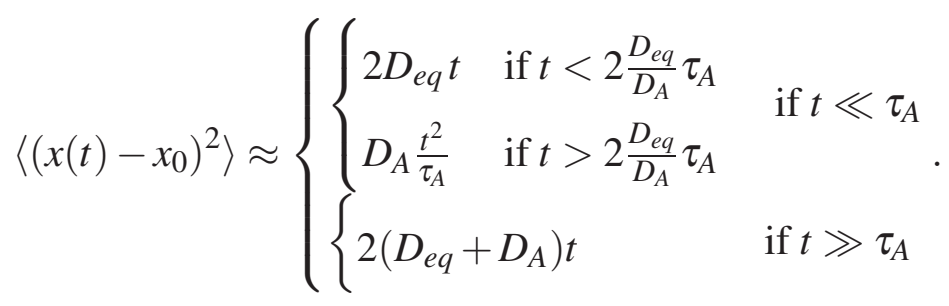

In the short-time limit, i.e., for $t \ll \tau_{A}$, the motion approaches a ballistic behavior when $t>2 \frac{D_{e q}}{D_{A}} \tau_{A}$, though at $t<2 \frac{D_{e q}}{D_{A}} \tau_{A}$, the dynamics is Fickian with the thermal diffusivity $D_{e q}$. On the other hand, the MSD for the long time limit $t \gg \tau_{A}$ is linear in time, clearly suggesting a diffusive regime with an elevated diffusivity. The results are graphically displayed in Fig. 2 and are equivalent to the one observed for the case in a homogeneous environment [80].

To know more about the dynamics, particularly the tail behavior, we compute the non-Gaussian parameter (NGP) defined as $\gamma_{G}=\frac{\left\langle x^{4}(t)\right\rangle}{3\left\langle x^{2}(t)\right\rangle^{2}}-1$, considering $x_{0}=0$, without any loss of information. For the switching diffusion, the NGP is given by (see the derivation in Appendix A 1)

$$
\gamma_{G}(t)=\frac{2\left(D_{2}-D_{1}\right)^{2}}{\left(r_{12}+r_{21}\right)^{4}} \frac{r_{12} r_{21}\left(\left(r_{12}+r_{21}\right) t-1+e^{-t\left(r_{12}+r_{21}\right)}\right)}{\left(D_{A}\left(t-\tau_{A}\right)+D_{e q} t+e^{-\frac{t}{\tau_{A}}} D_{A} \tau_{A}\right)^{2}}
$$


In the regime $t \ll \tau_{A}$, it approximates to

$$
\gamma_{G}(t) \approx \frac{2\left(D_{2}-D_{1}\right)^{2} r_{12} r_{21}\left(\left(r_{12}+r_{21}\right) t-1+e^{-t\left(r_{12}+r_{21}\right)}\right)}{\left(r_{12}+r_{21}\right)^{4}\left(D_{e q} t+\frac{1}{2} D_{A} \frac{t^{2}}{\tau_{A}}\right)^{2}}
$$

which implies $\gamma_{G}(t) \propto \tau_{A}^{2}$ at short times. This can be understood from Fig. 3 (a). In the extremely short-time limit $t \rightarrow 0$, or equivalently for $1 \gg r_{12} t, 1 \gg r_{21} t, t \ll \tau_{A}$, the NGP converges to a fixed value $\frac{\left(D_{2}-D_{1}\right)^{2} r_{12} r_{21}}{\left(r_{12}+r_{21}\right)^{2} D_{e q}^{2}}$, which is independent of $\tau_{A}$ and $D_{A}$, as can be seen in Figs. 3 (a) and 4 (a). This indicates that the particle was initially at a nonequilibrium state solely due to disorder. A quick check for the accuracy of our result is to take $D_{2}=D_{1}$, and notice that the NGP vanishes to zero at short times, which correctly reproduces the result for the case without disorder. As time passes, $\gamma_{G}(t)$ decreases monotonically, and it decays faster for small values of $\tau_{A}$ and large values of $D_{A}$, as illustrated in in Figs. 3 (a) and 4 (a). From Fig. 3 (c) one can also see that the NGP is a monotonically increasing function of $\tau_{A}$, the slope in the short $\tau_{A}$ limit gradually decreases as $t$ increases, though its effect at intermediate $\tau_{A}$ prevails only at a relevant timescale. In the long-time limit, the NGP can be approximated to

$$
\gamma_{G}(t) \approx \frac{2\left(D_{2}-D_{1}\right)^{2} r_{12} r_{21}}{\left(r_{12}+r_{21}\right)^{3} t\left(D_{e q}+D_{A}\right)^{2}}
$$

and it becomes zero at $t \rightarrow \infty$, implying the Gaussian behavior, as anticipated.

For the diffusing diffusivity model, the NGP is given by (for detailed calculation see A 2)

$$
\gamma_{G}(t)=\frac{D_{\mathrm{eq}}^{2}\left(2 t \omega-1+e^{-2 t \omega}\right)}{2\left(\omega t\left(D_{A}+D_{\mathrm{eq}}\right)-D_{A} \omega \tau_{A}+\omega D_{A} \tau_{A} e^{-\frac{t}{\tau_{A}}}\right)^{2}} .
$$

The NGP becomes unity and zero in the very short and large time limits, respectively. In the limit $t \ll \tau_{A}$, it can be approximated to

$$
\gamma_{G}(t) \approx \frac{D_{\mathrm{eq}}^{2}\left(2 t \omega-1+e^{-2 t \omega}\right)}{2 \omega^{2}\left(D_{\mathrm{eq}} t+\frac{1}{2} D_{A} t^{2} / \tau_{A}\right)^{2}}
$$

Like the previous case, the value of $\gamma_{G}(t)$ strongly depends on $\tau_{A}$ as well as $D_{A}$, as can be seen in panels (b) and (d) of Fig. 3 and in Fig. 4 (b). Also, similar dependence of $\omega$ on the NGP can be found, as shown in Fig. 3 (b). As the environment rearranges fast when $\omega$ is large, the motion is sampled over all realizations at a shorter timescale and consequently, the particle achieves a Gaussian distribution, which is reflected in the plot of $\gamma_{G}(t)$ shown in Fig. 3 (b). 

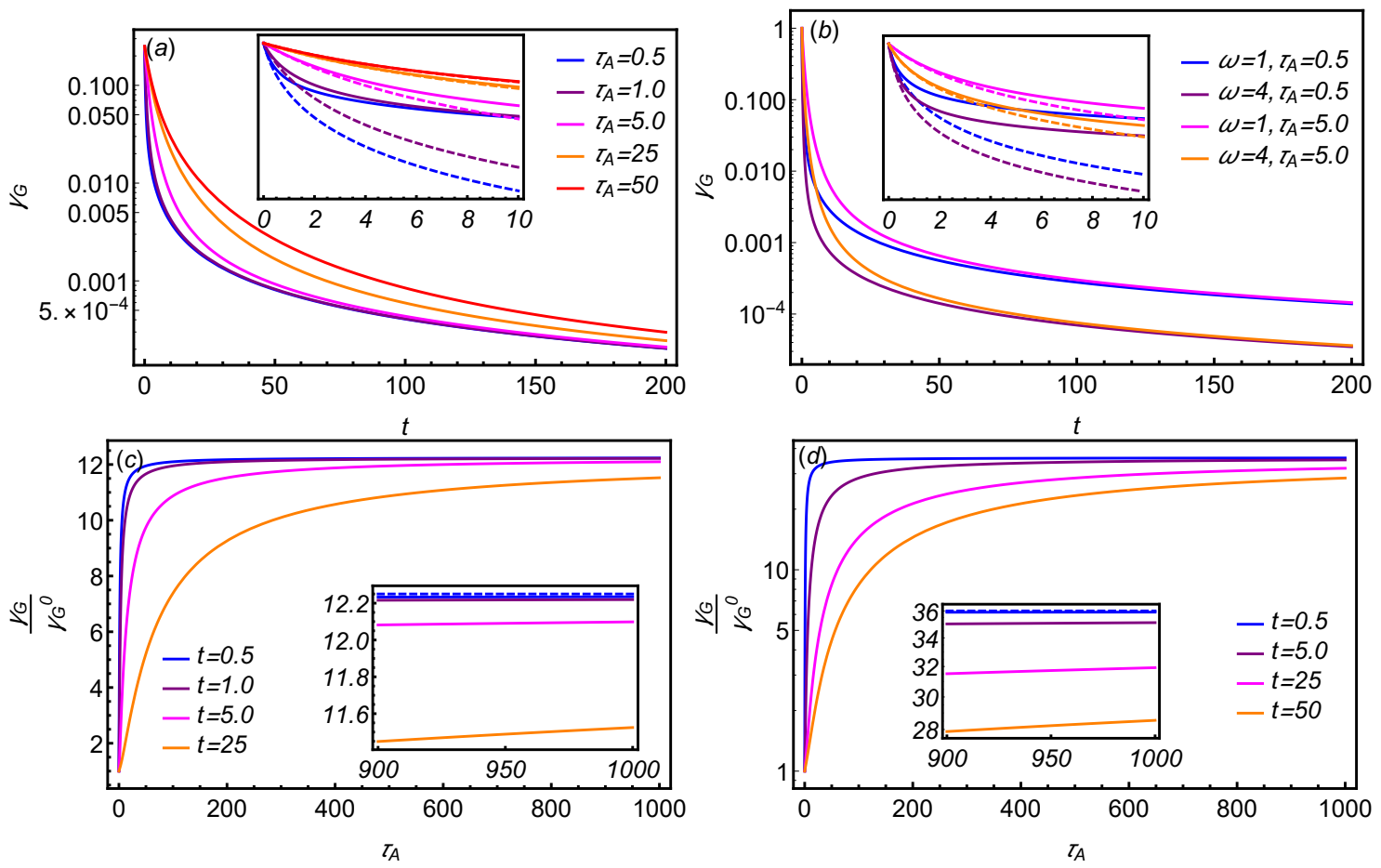

Figure 3. The non-Gaussian parameter (NGP) is plotted (i) as a function of time $t$ for different values of $\tau_{A}$ in panels (a)-(b), and (ii) the plots of NGP scaled by its value at $\tau_{A}=0$ [see Eqs. (A9) and (A16)] are shown in panels (c)-(d) as a function of the correlation time of the active noise $\tau_{A}$ at different times. In panels (a) and (c), the thermal diffusivity is modelled by the switching diffusion, and the curves correspond to Eq. (17). The parameters used here are given by the set $\left\{D_{1}=5, D_{2}=15, D_{A}=25, r_{12}=r_{21}=1 / 2\right\}$. In panels (b) and (d), the diffusing diffusivity model is used to describe the fluctuating diffusivity, and the curves are obtained using Eq. (20). Here, the values of other parameters are $D_{e q}=5, D_{A}=25$. For panel (d), we take $\omega=1.0$. In the insets of panels (a) and (b), the plots are compared with the approximate results for $t \ll \tau_{A}$ (represented by the dashed lines) given by Eqs. (18) and (21), respectively. The plots for large- $\tau_{A}$ limit are enlarged in insets of panels (c) and (d) to show that the ratio $\gamma_{G} / \gamma_{G}^{0}$ converges to a constant value [see Eq. [A10)] which is represented by the dashed lines.

Switching diffusion: Here we further investigate the dynamical properties by finding the complete PDF for the model of switching diffusion. By virtue of Eqs. (6) and (13), the PDF given in 

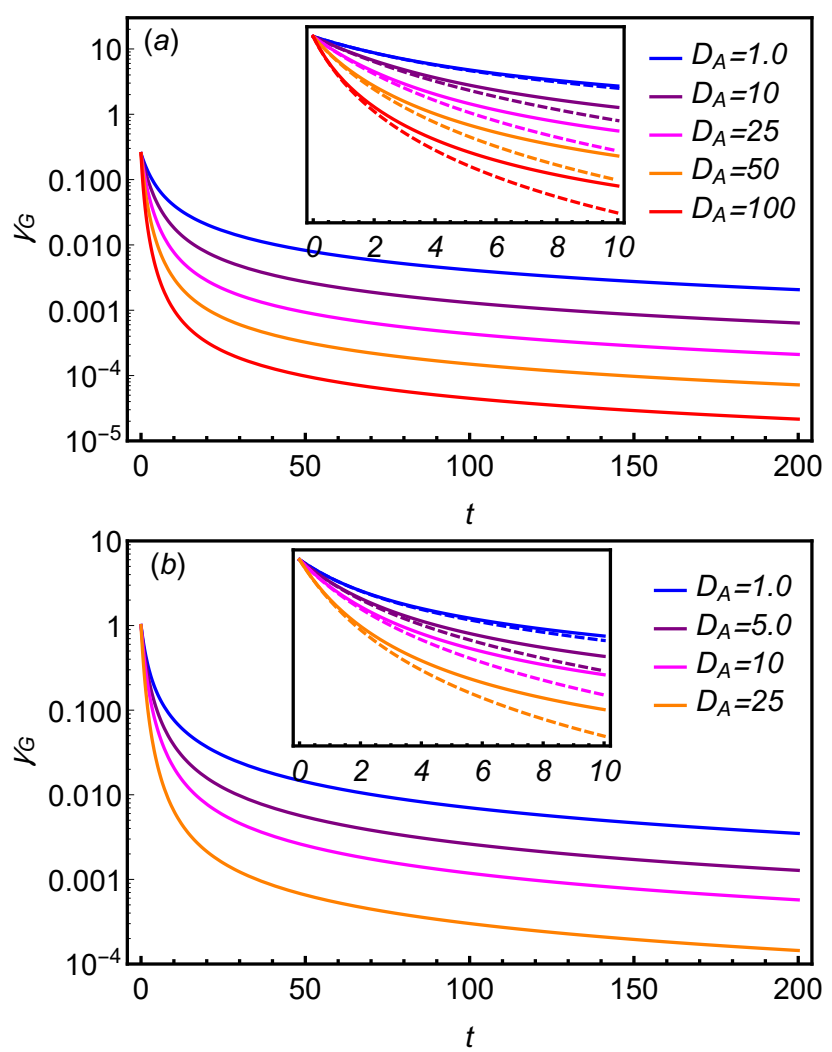

Figure 4. Logarithmic pots of the non-Gaussian parameter (NGP) as a function of time $t$ for different values of $D_{A}$ in the case of (a) switching diffusion and (b) diffusing diffusivity model. The short-time behavior is shown in the inset and can be well described by the approximate results (represented by dashed lines) given by Eqs. (18) and (21), respectively. For both models, we have taken $\tau_{A}=5$. The other parameters used in panel (a) are $D_{1}=5, D_{2}=15, r_{12}=r_{21}=\frac{1}{2}$, and in panel (b) these are $D_{e q}=5, \omega=1$.

Eq. (10) can be written explicitly as

$$
\begin{aligned}
& P\left(x, t \mid x_{0}=0,0\right) \\
& =\frac{1}{2 \pi} \int_{-\infty}^{\infty} d p e^{-i p x} \frac{1}{2}\left[1-\frac{\phi_{3}}{\phi_{2}}\right] e^{-\left(\phi_{1}+\phi_{2}\right) t} e^{-D_{A} p^{2}\left(t+\tau_{A}\left(e^{-\frac{t}{\tau_{A}}}-1\right)\right)} \\
& \quad+\frac{1}{2 \pi} \int_{-\infty}^{\infty} d p e^{-i p x} \frac{1}{2}\left[1+\frac{\phi_{3}}{\phi_{2}}\right] e^{-\left(\phi_{1}-\phi_{2}\right) t} e^{-D_{A} p^{2}\left(t+\tau_{A}\left(e^{-\frac{t}{\tau_{A}}}-1\right)\right)} .
\end{aligned}
$$

The integration in the above equation cannot be evaluated exactly. However, we can consider different limiting cases as demonstrated in Appendix B 1. At a timescale shorter than the inverse of switching rates, the distribution is the weighted average of two Gaussian functions centered at 
$x_{0}$, as given by (see Eq. (ㅍ1)

$$
P\left(x, t \mid x_{0}, 0\right)=p_{1, s} \frac{e^{-\frac{\left(x-x_{0}\right)^{2}}{2\left\langle x^{2}(t)\right\rangle_{1}}}}{\sqrt{2 \pi\left\langle x^{2}(t)\right\rangle_{1}}}+p_{2, s} \frac{e^{-\frac{\left(x-x_{0}\right)^{2}}{2\left\langle x^{2}(t)\right\rangle_{2}}}}{\sqrt{2 \pi\left\langle x^{2}(t)\right\rangle_{2}}},
$$

with $\left\langle x^{2}(t)\right\rangle_{1}=2\left[D_{1} t+D_{A}\left(t+\tau_{A}\left(e^{-\frac{t}{\tau_{A}}}-1\right)\right)\right],\left\langle x^{2}(t)\right\rangle_{2}=2\left[D_{2} t+D_{A}\left(t+\tau_{A}\left(e^{-\frac{t}{\tau_{A}}}-1\right)\right)\right]$ and $p_{i, s}$ being the steady-state probabilities defined in Eq. (5). So it shows a non-Gaussian behavior with a narrow central peak region which becomes pronounced at higher values of $\tau_{A}$ and lower values of $D_{A}$, as can be seen in Figs. 5 and 6 . These results are in consistent with the previous analysis on the non-Gaussian parameter, and it certainly requires a word of explanation. In our model, the dynamics solely driven by the active noise always shows a Gaussian behavior due to the Gaussian characteristics of the noise. However, with the introduction of dynamical disorder in the form of fluctuating thermal diffusivity, the system tends to deviate from the Gaussianity at short and intermediate timescales. Therefore, there is a competition between two opposing factors which determine the (non-)Gaussianity of the system. Naturally, prominent Gaussian behavior dictated by broader Gaussian central region prevails if the strength of the active noise given by the ratio $D_{A} / \tau_{A}$ becomes large. So using this argument all the previous results on the impact of $D_{A}$ and $\tau_{A}$ can be inferred. With the progress of time, the height of the peak decreases and the distribution flattens and eventually, it converges to a single Gaussian as illustrated in Fig. 7 . The Gaussian has the following form

$$
P\left(x, t \mid x_{0}=0,0\right)=\frac{e^{-\frac{x^{2}}{2\left\langle x^{2}(t)\right\rangle_{e q}}}}{\sqrt{2 \pi\left\langle x^{2}(t)\right\rangle_{e q}}}
$$

with $\left\langle x^{2}(t)\right\rangle_{e q}=2\left[D_{e q} t+D_{A}\left(t+\tau_{A}\left(e^{-\frac{t}{\tau_{A}}}-1\right)\right)\right]$. The calculations for this limiting case has been given in Appendix B 1. The above result is not very surprising as in the long-time limit the particle explores all possible realizations with different diffusivities, thereby displaying Gaussian properties with an equilibrium diffusivity. Unlike the passive-particle case, here the width $\left\langle x^{2}(t)\right\rangle_{e q}$ is enhanced, and it is expressed in terms of the equilibrium thermal diffusivity and active diffusivity. Note that here the pattern of convergence to a Gaussian is strikingly different from the one observed in a system with strong static disorder where the peak narrows down to a single point in the long time [81]. Our model is related to the one in Ref. [69] where a soft colloid diffusing in response to the gradients in chemical potential and/or concentrations switches between two states with different masses due to active interactions. Unlike Ref. [69], here we consider the motion of 


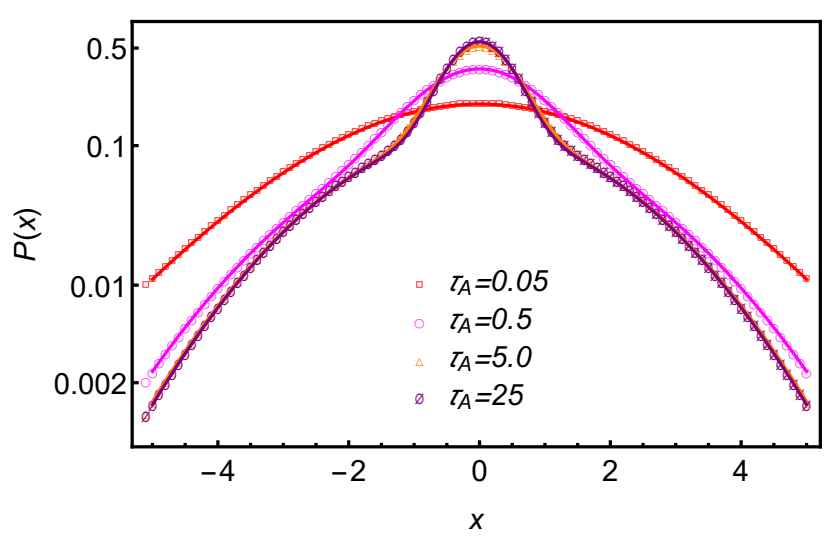

Figure 5. Active particle with switching diffusion: spatial distribution computed numerically from Eq. (22) is plotted with symbols as a function of displacement $x$ for different values of $\tau_{A}$ at a short time $t=0.10$. The values of other parameters are as follows: $D_{1}=1, D_{2}=14, r_{12}=1 / 2, r_{21}=1 / 2, D_{A}=25$. The solid lines which correspond to the approximate result given in Eq. (23) are in good agreement with the numerical ones.



Figure 6. Active particle with switching diffusion: PDF versus displacement plot for $t=0.50$ and $\tau_{A}=5.0$. The values of other parameters $\left(D_{1}, D_{2}, r_{12}, r_{21}\right)$ are same as in Fig. 5, and the solid lines representing the approximate result [Eq. 23)] are drawn for the purpose of comparison like Fig. 5

a soft active colloid, which means that the dynamics is not only driven by the chemical or/and concentration gradient, but it also moves due to self-propulsion mechanism fueled by ATP hydrolysis. However, it is interesting to note that both studies capture the non-Gaussian behavior at a shorter timescale, which further emphasizes the fact that the non-Gaussianity is a very common feature associated with a disordered system. 

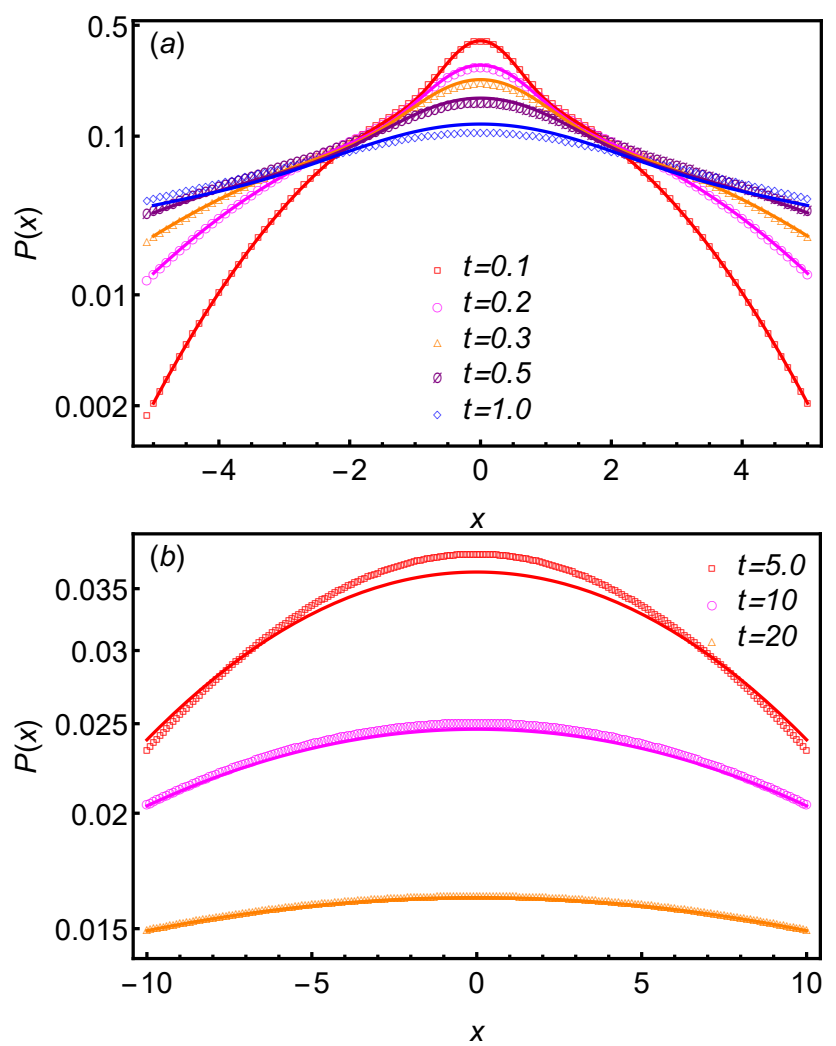

Figure 7. Probability distribution function versus displacement in logarithmic scale at different times for the case where the thermal diffusivity switches between two values $D_{1}$ (state 1 ) and $D_{2}$ (state 2) at Poisson rates (from state 2 to 1) $r_{21}$ and (vice versa) $r_{12}$. The curves with symbols are obtained numerically using Eq. (22) by taking the set of parameters as follows: $\left\{D_{1}=1, D_{2}=14, r_{12}=3 / 4, r_{21}=1 / 4, \tau_{A}=50, D_{A}=25\right\}$. In panel (a) the distribution is for small times and is approximately similar to Eq. (23) as depicted by solid lines. In panel (b) the solid lines correspond to Eq. (24) and match well with the numerical results in the long-time limit.

Diffusing diffusivity model: $\quad$ From Eq. (10), with aid of Eqs. (13) and (7), the spatial distribution can be expressed as

$$
\begin{aligned}
& P\left(x, t \mid x_{0}=0,0\right) \\
& =\frac{1}{2 \pi} \int_{-\infty}^{\infty} d p e^{-i p x} \frac{4 \omega \beta e^{-(\beta-\omega) t}}{(\beta+\omega)^{2}-(\beta-\omega)^{2} e^{-2 \beta t}} e^{-D_{A} p^{2}\left(t+\tau_{A}\left(e^{-\frac{t}{\tau_{A}}}-1\right)\right)} .
\end{aligned}
$$

The above integration is evaluated numerically and plotted in Figs. 8 and 9 . Unlike the switching diffusion case, the distribution at short times is expressed by double exponential functions of the 
form

$$
\begin{aligned}
& P\left(x, t \mid x_{0}=0,0\right)
\end{aligned}
$$

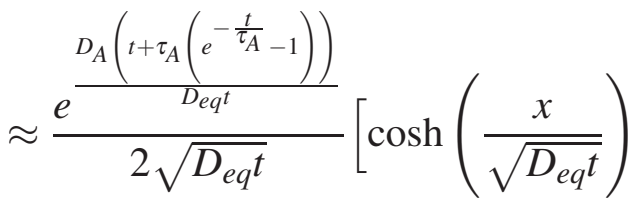

$$
\begin{aligned}
& \left.-\operatorname{erf}\left(\frac{x}{\sqrt{\frac{1}{4\left[D_{A}\left(t+\tau_{A}\left(e^{-\frac{t}{\tau_{A}}}-1\right)\right)\right]}}}\right) \sinh \left(\frac{x}{\sqrt{D_{e q} t}}\right)\right] \text {, }
\end{aligned}
$$

which is also non-Gaussian in nature. See the derivation in Appendix B 2, The non-Gaussian nature at short times can also be observed from Fig. 8 (a). It is clear from Fig. 9 that the distribution for large values of $\tau_{A}$ has a significantly long exponential tail, which indirectly suggests that the long persistence strongly enhances the effect of disorder on the particle's motion. On the other hand, the nature of non-Gaussianity diminishes as the strength of active noise increases, as can be comprehended from Fig. 10. This is also complemented by the previous NGP analysis. Similar explanations as presented in the case of switching diffusion can be given to understand such properties in the intermediate-time regime, namely, with increasing the strength of active noise the Gaussian properties prevail. As time passes, the height of the distribution gets reduced with flattening of the curve as shown in Fig. 8 (b), and after a long time, the PDF becomes a Gaussian distribution given by Eq. (B4).

\section{CONCLUSION}

In this paper, we have investigated the dynamics of a single active Ornstein-Uhlenbeck particle (OUP) subjected to the dynamical disorder. Invoking the idea of fluctuating thermal diffusivity, we have shown that the distribution becomes non-Gaussian at intermediate times, which cannot be found in the usual OUP model, but the behavior is not very surprising as this is the signature of a disordered system. However, unlike the passive disordered case, the dynamics is not always Fickian; we have observed a ballistic behavior at a timescale shorter than its persistence time, signifying the directed motion of the active particle. Also it has been found that the non-Gaussianity in the distribution is strongly influenced by the strength of the active noise suggesting that the longer correlation arising due to activity facilitates the effect of disorder on the dynamics. 

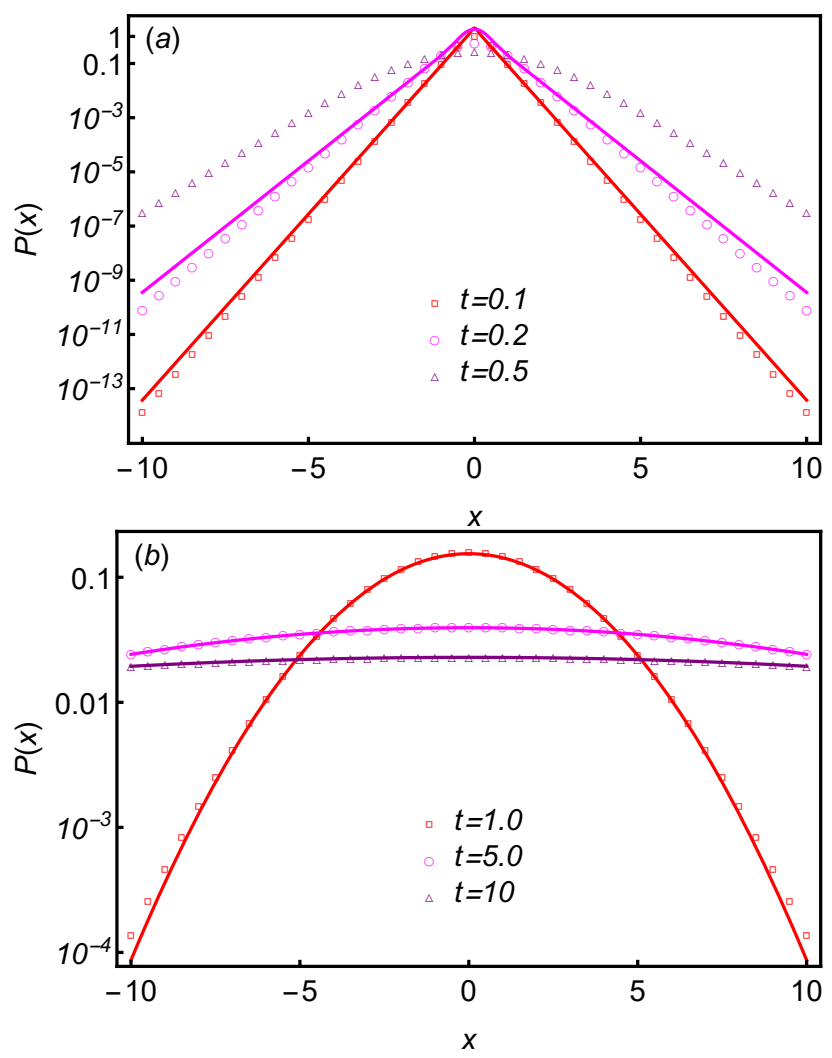

Figure 8. Logarithmic plot of probability distribution function at different times as a function of displacement considering that the thermal diffusivity $D(t)$ is a stochastic variable following the OU process. The characteristic timescales for thermal diffusivity and active noise are $1 / \omega=0.4, \tau_{A}=5.0$, respectively. The numerical results obtained from Eq. (25) are plotted using symbols taking $D_{A}=25$. In panel (a) the plots are for small times, and the solid lines correspond to the analytical result given by Eq. (26). In panel (b) the solid lines correspond to Eq. (B4).

The extensions of this work may be manyfold: In one direction, one can explore the effect of heterogeneity on several properties of a non-interacting active system which are at certain extent studied in the context of passive particle. In other direction, it can be extended to higher dimensions which is relevant while to be dealing with real biological systems. In addition, one may incorporate heterogeneity and interactions together to look into the many-body problem such as biopolymers and active gels [82, 83].

\section{CONFLICTS OF INTEREST}

There are no conflicts to declare. 


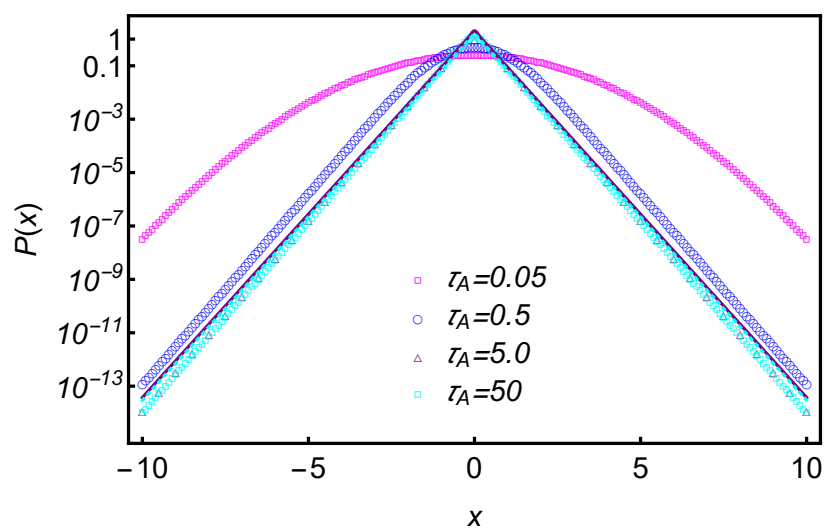

Figure 9. Active particle in a heterogeneous medium characterized by the diffusing diffusivity model: logarithmic plots of the spatial distribution numerically obtained from Eq. (25) and marked by symbols are compared with the analytical solution [Eq. 26] ] represented by the solid curves for different values of $\tau_{A}$ at a short time $t=0.10$. The values of other parameters are same as in Fig. 8 ,

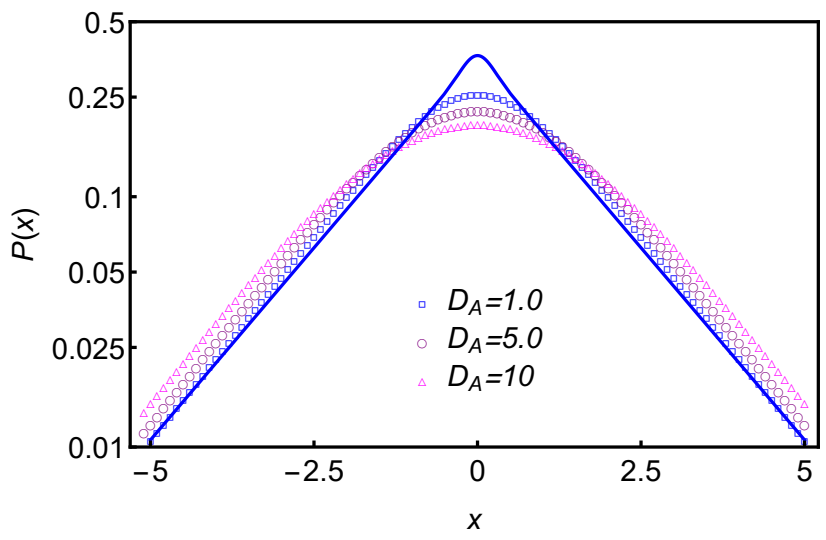

Figure 10. Active particle in a heterogeneous medium characterized by the diffusing diffusivity model: Like Fig. 9 the numerical plots of PDF are compared with the approximate distribution (solid curve) given by Eq. (26). The plots are for different values of $D_{A}$ at time $t=0.40$ and $\tau_{A}=1.0$. Other parameters are $D_{e q}=5$ and $\omega=1.0$.

\section{ACKNOWLEDGEMENTS}

R. C. acknowledges SERB for funding (Project No. MTR/2020/000230 under MATRICS scheme) and IRCC-IIT Bombay (Project No. RD/0518-IRCCAW0-001) for funding. K.G. acknowledges IIT Bombay for support through the institute postdoctoral fellowship. 


\section{Appendix A: Computation of moments and non-Gaussian parameter}

The second moment of the distribution as given by Eq. (15) can be computed using Eqs. (2) and (4), and it reads

$$
\begin{aligned}
& \left\langle\left(x(t)-x_{0}\right)^{2}\right\rangle \\
& =\int_{0}^{t} \int_{0}^{t} d t_{1} d t_{2}\left\langle\eta_{T}\left(t_{1}\right) \eta_{T}\left(t_{2}\right)\right\rangle+\int_{0}^{t} \int_{0}^{t} d t_{1} d t_{2}\left\langle v_{A}\left(t_{1}\right) v_{A}\left(t_{2}\right)\right\rangle \\
& =2 \int_{0}^{t} \int_{0}^{t} d t_{1} d t_{2}\left\langle D_{T}\left(t_{1}\right)\right\rangle \delta\left(t_{1}-t_{2}\right)+\frac{D_{A}}{\tau_{A}} \int_{0}^{t} \int_{0}^{t} d t_{1} d t_{2} e^{-\frac{\left|t_{1}-t_{2}\right|}{\tau_{A}}} \\
& =2\left\langle D_{T}(t)\right\rangle \int_{0}^{t} d t_{1}+2 D_{A} \int_{0}^{t} d t_{1}\left(1-e^{-\frac{t_{1}}{\tau_{A}}}\right) \\
& =2 D_{e q} t+2 D_{A}\left(t+\tau_{A}\left(e^{-\frac{t}{\tau_{A}}}-1\right)\right) .
\end{aligned}
$$

The fourth moment given in Eq. (11) can be expressed using Eq. (8), as follows:

$$
\begin{aligned}
& \left\langle\left(x(t)-x_{0}\right)^{4}\right\rangle \\
& =\int_{0}^{t} \int_{0}^{t} \int_{0}^{t} \int_{0}^{t} d t_{1} d t_{2} d t_{3} d t_{4}\left\langle\eta_{T}\left(t_{1}\right) \eta_{T}\left(t_{2}\right) \eta_{T}\left(t_{3}\right) \eta_{T}\left(t_{4}\right)\right\rangle \\
& +2 \int_{0}^{t} \int_{0}^{t} \int_{0}^{t} \int_{0}^{t} d t_{1} d t_{2} d t_{3} d t_{4}\left\langle\eta_{T}\left(t_{1}\right) \eta_{T}\left(t_{2}\right)\right\rangle\left\langle v_{A}\left(t_{3}\right) v_{A}\left(t_{4}\right)\right\rangle \\
& +\int_{0}^{t} \int_{0}^{t} \int_{0}^{t} \int_{0}^{t} d t_{1} d t_{2} d t_{3} d t_{4}\left\langle v_{A}\left(t_{1}\right) v_{A}\left(t_{2}\right) v_{A}\left(t_{3}\right) v_{A}\left(t_{4}\right)\right\rangle .
\end{aligned}
$$

Now, with the aid of Eq. (11) and the autocorrelation function of $v_{A}(t)$ as given in Eq. (4), the last term of the RHS in the above equation can be computed, and it reads

$$
\begin{aligned}
& \int_{0}^{t} \int_{0}^{t} \int_{0}^{t} \int_{0}^{t} d t_{1} d t_{2} d t_{3} d t_{4}\left\langle v_{A}\left(t_{1}\right) v_{A}\left(t_{2}\right) v_{A}\left(t_{3}\right) v_{A}\left(t_{4}\right)\right\rangle \\
& =\int_{0}^{t} \int_{0}^{t} d t_{1} d t_{2}\left\langle v_{A}\left(t_{1}\right) v_{A}\left(t_{2}\right)\right\rangle \int_{0}^{t} \int_{0}^{t} d t_{3} d t_{4}\left\langle v_{A}\left(t_{3}\right) v_{A}\left(t_{4}\right)\right\rangle \\
& +\int_{0}^{t} \int_{0}^{t} d t_{1} d t_{3}\left\langle v_{A}\left(t_{1}\right) v_{A}\left(t_{3}\right)\right\rangle \int_{0}^{t} \int_{0}^{t} d t_{2} d t_{4}\left\langle v_{A}\left(t_{2}\right) v_{A}\left(t_{4}\right)\right\rangle \\
& +\int_{0}^{t} \int_{0}^{t} d t_{1} d t_{4}\left\langle v_{A}\left(t_{1}\right) v_{A}\left(t_{4}\right)\right\rangle \int_{0}^{t} \int_{0}^{t} d t_{2} d t_{3}\left\langle v_{A}\left(t_{2}\right) v_{A}\left(t_{3}\right)\right\rangle \\
& =3\left[\frac{D_{A}}{\tau_{A}} \int_{0}^{t} \int_{0}^{t} d t_{1} d t_{2} e^{-\frac{\left|t_{1}-t_{2}\right|}{\tau_{A}}} \times \frac{D_{A}}{\tau_{A}} \int_{0}^{t} \int_{0}^{t} d t_{3} d t_{4} e^{-\frac{\left|t_{3}-t_{4}\right|}{\tau_{A}}}\right] \\
& =12\left[D_{A}\left(\tau_{A}\left(e^{-\frac{t}{\tau_{A}}}-1\right)+t\right)\right]^{2} .
\end{aligned}
$$


Using the properties of white noise $\eta_{T}(t)$, the first term of the RHS in Eq. (A2) can be written as

$$
\begin{aligned}
& \int_{0}^{t} \int_{0}^{t} \int_{0}^{t} \int_{0}^{t} d t_{1} d t_{2} d t_{3} d t_{4}\left\langle\eta_{T}\left(t_{1}\right) \eta_{T}\left(t_{2}\right) \eta_{T}\left(t_{3}\right) \eta_{T}\left(t_{4}\right)\right\rangle \\
& =\int_{0}^{t} \int_{0}^{t} \int_{0}^{t} \int_{0}^{t} d t_{1} d t_{2} d t_{3} d t_{4}\left\langle\left\langle\eta_{T}\left(t_{1}\right) \eta_{T}\left(t_{2}\right)\right\rangle\left\langle\eta_{T}\left(t_{3}\right) \eta_{T}\left(t_{4}\right)\right\rangle\right\rangle_{D_{T}} \\
& +\int_{0}^{t} \int_{0}^{t} \int_{0}^{t} \int_{0}^{t} d t_{1} d t_{3} d t_{2} d t_{4}\left\langle\left\langle\eta_{T}\left(t_{1}\right) \eta_{T}\left(t_{3}\right)\right\rangle\left\langle\eta_{T}\left(t_{2}\right) \eta_{T}\left(t_{4}\right)\right\rangle\right\rangle_{D_{T}} \\
& +\int_{0}^{t} \int_{0}^{t} \int_{0}^{t} \int_{0}^{t} d t_{1} d t_{4} d t_{2} d t_{3}\left\langle\left\langle\eta_{T}\left(t_{1}\right) \eta_{T}\left(t_{4}\right)\right\rangle\left\langle\eta_{T}\left(t_{2}\right) \eta_{T}\left(t_{3}\right)\right\rangle\right\rangle_{D_{T}} \\
& =24 \int_{0}^{t} d t_{1} \int_{0}^{t_{1}} d t_{2}\left\langle D_{T}\left(t_{1}\right) D_{T}\left(t_{2}\right)\right\rangle .
\end{aligned}
$$

The middle term of the RHS in Eq. (A2) is given by

$$
\begin{aligned}
& \int_{0}^{t} \int_{0}^{t} \int_{0}^{t} \int_{0}^{t} d t_{1} d t_{2} d t_{3} d t_{4}\left\langle\eta_{T}\left(t_{1}\right) \eta_{T}\left(t_{2}\right)\right\rangle\left\langle v_{A}\left(t_{3}\right) v_{A}\left(t_{4}\right)\right\rangle \\
& =12 t \int_{0}^{t} d t_{1} \int_{0}^{t_{1}} d t_{2}\left\langle D\left(t_{1}\right)\right\rangle\left\langle v_{A}\left(t_{1}\right) v_{A}\left(t_{2}\right)\right\rangle
\end{aligned}
$$

For the two models of $D_{T}(t)$, we solve Eqs. (A4) and (A5). The calculations are shown below.

\section{Switching diffusion}

To perform the integration in Eqs. (A4) and (A5), it is required to know the average and autocorrelation function of $D(t)$ which are given by [84] $\langle D(t)\rangle=\frac{r_{12} D_{2}+r_{21} D_{1}}{r_{12}+r_{21}}$, and $\left\langle D\left(t_{1}\right) D\left(t_{2}\right)\right\rangle=$ $\left\langle D\left(t_{1}\right)\right\rangle^{2}+\frac{r_{12} r_{21}}{\left(r_{12}+r_{21}\right)^{2}}\left(D_{2}-D_{1}\right)^{2} e^{-\left(r_{12}+r_{21}\right)\left|t_{2}-t_{1}\right|}$. Using these, one can compute Eq. A4 as

$$
\begin{gathered}
\int_{0}^{t} \int_{0}^{t} \int_{0}^{t} \int_{0}^{t} d t_{1} d t_{2} d t_{3} d t_{4}\left\langle\eta_{T}\left(t_{1}\right) \eta_{T}\left(t_{2}\right) \eta_{T}\left(t_{3}\right) \eta_{T}\left(t_{4}\right)\right\rangle \\
=24 \int_{0}^{t} d t_{1} \int_{0}^{t_{1}} d t_{2}\left[\left(\frac{r_{12} D_{2}+r_{21} D_{1}}{r_{12}+r_{21}}\right)^{2}\right. \\
\left.\quad+\frac{r_{12} r_{21}}{\left(r_{12}+r_{21}\right)^{2}}\left(D_{2}-D_{1}\right)^{2} e^{-\left(r_{12}+r_{21}\right)\left(t_{1}-t_{2}\right)}\right] \\
=\frac{12}{\left(r_{12}+r_{21}\right)^{3}}\left[\left(r_{12}+r_{21}\right) t^{2}\left(D_{2} r_{12}+D_{1} r_{21}\right)^{2}\right. \\
\left.+2\left(D_{1}-D_{2}\right)^{2} r_{12} r_{21}\left(\frac{e^{\left(-r_{12}-r_{21}\right) t}-1}{r_{12}+r_{21}}+t\right)\right] .
\end{gathered}
$$


Similarly, one has

$$
\begin{aligned}
& 2 \int_{0}^{t} \int_{0}^{t} \int_{0}^{t} \int_{0}^{t} d t_{1} d t_{2} d t_{3} d t_{4}\left\langle\eta_{T}\left(t_{1}\right) \eta_{T}\left(t_{2}\right)\right\rangle\left\langle v_{A}\left(t_{3}\right) v_{A}\left(t_{4}\right)\right\rangle \\
& =\frac{24 D_{A} t\left(D_{2} r_{12}+D_{1} r_{21}\right) e^{-\frac{t}{\tau_{A}}}\left(e^{t / \tau_{A}}\left(t-\tau_{A}\right)+\tau_{A}\right)}{r_{12}+r_{21}}
\end{aligned}
$$

By virtue of Eqs. (A3), (A6), (A7) and (15), the NGP defined by $\gamma_{G}=\frac{\left\langle x^{4}(t)\right\rangle}{3\left\langle x^{2}(t)\right\rangle^{2}}-1$ can be calculated, and it yields

$$
\begin{aligned}
& \gamma_{G}(t)=\frac{2\left(D_{2}-D_{1}\right)^{2}}{\left(r_{12}+r_{21}\right)^{2}} \times \\
& \frac{r_{12} r_{21}\left(\left(r_{12}+r_{21}\right) t-1+e^{-t\left(r_{12}+r_{21}\right)}\right)}{\left(\left(r_{12}+r_{21}\right) D_{A}\left(t-\tau_{A}\right)+\left(D_{2} r_{12}+D_{1} r_{21}\right) t+e^{-\frac{t}{\tau_{A}}}\left(r_{12}+r_{21}\right) D_{A} \tau_{A}\right)^{2}}
\end{aligned}
$$

For $\tau_{A}=0$, the NGP denoted here as $\gamma_{G}^{0}(t)$ can be expressed as

$$
\gamma_{G}^{0}(t)=\frac{2\left(D_{2}-D_{1}\right)^{2} r_{12} r_{21}\left(\left(r_{12}+r_{21}\right) t-1+e^{-t\left(r_{12}+r_{21}\right)}\right)}{\left(r_{12}+r_{21}\right)^{4} t^{2}\left(D_{A}+D_{e q}\right)^{2}} .
$$

For $\tau_{A} \rightarrow \infty$ and finite values of $t$, the ratio $\gamma_{G}(t) / \gamma_{G}^{0}(t)$ takes a constant value, viz.,

$$
\frac{\gamma_{G}(t)}{\gamma_{G}^{0}(t)} \approx\left(1+\frac{D_{A}}{D_{e q}}\right)^{2}
$$

\section{Diffusing diffusivity model}

Using the dynamical equation of $\xi(t)$ given in Sec. I B 2, one can write

$$
\begin{aligned}
& \left\langle\left(\xi\left(t_{1}\right)-\xi(0) e^{-\omega t_{1}}\right)\left(\xi\left(t_{2}\right)-\xi(0) e^{-\omega t_{2}}\right)\right\rangle \\
& =\left\langle\xi\left(t_{1}\right) \xi\left(t_{2}\right)\right\rangle \\
& -\left\langle\xi\left(t_{1}\right) \xi(0)\right\rangle e^{-\omega t_{2}}-\left\langle\xi\left(t_{2}\right) \xi(0)\right\rangle e^{-\omega t_{1}}+\langle\xi(0) \xi(0)\rangle e^{-\omega t_{1}-\omega t_{2}} \\
& =\int_{0}^{t_{1}} \int_{0}^{t_{2}} d t_{1}^{\prime} d t_{2}^{\prime} e^{-\omega\left(t_{1}-t_{1}^{\prime}\right)} e^{-\omega\left(t_{2}-t_{2}^{\prime}\right)}\left\langle\eta_{A}\left(t_{1}^{\prime}\right) \eta_{A}\left(t_{2}^{\prime}\right)\right\rangle,
\end{aligned}
$$

which implies $\left\langle\xi\left(t_{1}\right) \xi\left(t_{2}\right)\right\rangle=\left\langle\xi^{2}(0)\right\rangle e^{-\omega\left|t_{1}-t_{2}\right|}$. With these, one can get

$$
\begin{aligned}
\sum_{i, j=1}^{2}\left\langle\xi_{i}^{2}\left(t_{1}\right) \xi_{j}^{2}\left(t_{2}\right)\right\rangle & =\sum_{i, j=1, i \neq j}^{2}\left\langle\xi_{i}^{2}\left(t_{1}\right)\right\rangle\left\langle\xi_{j}^{2}\left(t_{2}\right)\right\rangle+\sum_{i=1}^{2}\left\langle\xi_{i}^{2}\left(t_{1}\right) \xi_{i}^{2}\left(t_{2}\right)\right\rangle \\
& =2\left\langle\xi^{2}(0)\right\rangle^{2}+\sum_{i=1}^{2}\left(\left\langle\xi_{i}^{2}\left(t_{1}\right)\right\rangle^{2}+2\left\langle\xi_{i}\left(t_{1}\right) \xi_{i}\left(t_{2}\right)\right\rangle^{2}\right) \\
& =4\left\langle\xi^{2}(0)\right\rangle^{2}+4\left\langle\xi^{2}(0)\right\rangle^{2} e^{-2 \omega\left|t_{1}-t_{2}\right|} .
\end{aligned}
$$


Here, $\langle D(t)\rangle=\sum_{n=1}^{2} \xi_{n}^{2}(t)$, and $\left\langle\xi_{i}^{2}(t)\right\rangle=\left\langle\xi_{i}^{2}(0)\right\rangle=\frac{1}{2} D_{e q}$. Using Eq. (A12), Eq. (A4) can be calculated as follows:

$$
\begin{aligned}
& \int_{0}^{t} \int_{0}^{t} \int_{0}^{t} \int_{0}^{t} d t_{1} d t_{2} d t_{3} d t_{4}\left\langle\eta_{T}\left(t_{1}\right) \eta_{T}\left(t_{2}\right) \eta_{T}\left(t_{3}\right) \eta_{T}\left(t_{4}\right)\right\rangle \\
& =24 \int_{0}^{t} d t_{1} \int_{0}^{t_{1}} d t_{2}\left\langle D_{T}\left(t_{1}\right) D_{T}\left(t_{2}\right)\right\rangle \\
& =24 \int_{0}^{t} d t_{1} \int_{0}^{t_{1}} d t_{2} \sum_{i, j=1}^{2}\left\langle\xi_{i}^{2}\left(t_{1}\right) \xi_{j}^{2}\left(t_{2}\right)\right\rangle \\
& =24 \int_{0}^{t} d t_{1} \int_{0}^{t_{1}} d t_{2} \frac{1}{4}\left\langle\xi^{2}\left(t_{1}\right)\right\rangle^{2}+24 \int_{0}^{t} d t_{1} \int_{0}^{t_{1}} d t_{2} \frac{1}{4}\left\langle\xi^{2}(0)\right\rangle^{2} e^{-2 \omega\left|t_{1}-t_{2}\right|} \\
& =\frac{6 D_{\mathrm{eq}}^{2}\left[2 t \omega(t \omega+1)+e^{-2 t \omega}-1\right]}{\omega^{2}} .
\end{aligned}
$$

Similarly, from Eq. A5 one can obtain

$$
\begin{aligned}
& 2 \int_{0}^{t} \int_{0}^{t} \int_{0}^{t} \int_{0}^{t} d t_{1} d t_{2} d t_{3} d t_{4}\left\langle\eta_{T}\left(t_{1}\right) \eta_{T}\left(t_{2}\right)\right\rangle\left\langle v_{A}\left(t_{3}\right) v_{A}\left(t_{4}\right)\right\rangle \\
& =24 t \int_{0}^{t} d t_{1} \int_{0}^{t_{1}} d t_{2}\left\langle D\left(t_{1}\right)\right\rangle\left\langle v_{A}\left(t_{1}\right) v_{A}\left(t_{2}\right)\right\rangle \\
& =24 D_{e q} D_{A} t\left(\tau_{A}\left(e^{-\frac{t}{\tau_{A}}}-1\right)+t\right) .
\end{aligned}
$$

Using Eqs. (A3), (A13), (A14) and (15), the NGP can be calculated, and it reads

$$
\gamma_{G}(t)=\frac{\left\langle x^{4}(t)\right\rangle}{3\left\langle x^{2}(t)\right\rangle^{2}}-1=\frac{D_{\mathrm{eq}}^{2}\left(2 t \omega-1+e^{-2 t \omega}\right)}{2\left(\omega t\left(D_{A}+D_{\mathrm{eq}}\right)-D_{A} \omega \tau_{A}+\omega D_{A} \tau_{A} e^{-\frac{t}{\tau_{A}}}\right)^{2}} .
$$

From the above equation one can find the NGP at $\tau_{A}=0$, which reads

$$
\gamma_{G}^{0}(t)=\frac{\left\langle x^{4}(t)\right\rangle}{3\left\langle x^{2}(t)\right\rangle^{2}}-1=\frac{D_{\mathrm{eq}}^{2}\left(2 t \omega-1+e^{-2 t \omega}\right)}{2 \omega^{2} t^{2}\left(D_{A}+D_{\mathrm{eq}}\right)^{2}}
$$

Like the previous case, the ratio $\gamma_{G}(t) / \gamma_{G}^{0}(t)$ for $\tau_{A} \rightarrow \infty$ and finite values of $t$ is well approximated to Eq. (A10).

\section{Appendix B: Limiting values of PDF}

Here we show the calculation of approximate PDFs at different limits. Two models of the fluctuating thermal diffusivity are considered for the analysis. 


\section{Switching diffusion}

Here one can assign two diffusive timescales $D_{2} p^{2}$ and $D_{1} p^{2}$, to compare them with the switching rates $r_{12}$ and $r_{21}$. For $\frac{D_{2}}{r_{21}} p^{2} \gg 1$ and $\frac{D_{1}}{r_{12}} p^{2} \gg 1$, one gets $\phi_{2} \approx \frac{1}{2}\left[\left(D_{2}-D_{1}\right) p^{2}+\left(r_{21}-r_{12}\right)\right]+$ $\frac{r_{12} r_{21}}{\left(D_{2}-D_{1}\right) p^{2}+\left(r_{21}-r_{12}\right)}$. Clearly, it corresponds to the liming case at shorter times. With the above approximation, Eq. (22) modifies to

$$
\begin{aligned}
& P\left(x, t \mid x_{0}, 0\right) \\
& =\frac{1}{2 \pi} \int_{-\infty}^{\infty} d p e^{-i p\left(x-x_{0}\right)} \frac{r_{12}}{r_{12}+r_{21}} e^{-D_{2} p^{2} t} e^{-D_{A} p^{2}\left(t+\tau_{A}\left(e^{-\frac{t}{\tau_{A}}}-1\right)\right)} \\
& +\frac{1}{2 \pi} \int_{-\infty}^{\infty} d p e^{-i p\left(x-x_{0}\right)} \frac{r_{21}}{r_{12}+r_{21}} e^{-D_{1} p^{2} t} e^{-D_{A} p^{2}\left(t+\tau_{A}\left(e^{-\frac{t}{\tau_{A}}}-1\right)\right)} \\
& =\frac{r_{12}}{r_{12}+r_{21}} \frac{e^{-\frac{\left(x-x_{0}\right)^{2}}{4\left[D_{2} t+D_{A}\left(t+\tau_{A}\left(e^{-\frac{t}{\tau_{A}}}-1\right)\right)\right]}}}{\sqrt{4 \pi\left[D_{2} t+D_{A}\left(t+\tau_{A}\left(e^{-\frac{t}{\tau_{A}}}-1\right)\right)\right]}} \\
& +\frac{r_{21}}{r_{12}+r_{21}} \frac{e^{-\frac{\left(x-x_{0}\right)^{2}}{4\left[D_{1} t+D_{A}\left(t+\tau_{A}\left(e^{-\frac{t}{\tau_{A}}}-1\right)\right)\right]}}}{\sqrt{4 \pi\left[D_{1} t+D_{A}\left(t+\tau_{A}\left(e^{-\frac{t}{\tau_{A}}}-1\right)\right)\right]}} \text {. }
\end{aligned}
$$

Notice that, within the short-time regime one can consider two limiting cases, $r_{12} \gg r_{21}$ and $r_{12} \ll r_{21}$. For $r_{12} \gg r_{21}$, the first term on the RHS in Eq. (B1D dominates and the second term can be ignored, and therefore, the distribution becomes almost Gaussian with a width containing only $D_{2}$ and $D_{A}$. In the other limiting case, the behavior is mostly dictated by the second term. In the long-time limit, i.e., for $\frac{D_{2}}{r_{21}} p^{2} \ll 1$ and $\frac{D_{1}}{r_{12}} p^{2} \ll 1$, the following approximations can be done, $\phi_{2} \approx \frac{1}{2}\left(r_{12}+r_{21}\right)-\frac{p^{2}}{2} \frac{r_{12}-r_{21}}{r_{12}+r_{21}}\left(D_{2}-D_{1}\right)$, and $\phi_{1}-\phi_{2} \approx p^{2}\left(\frac{r_{12}}{r_{12}+r_{21}} D_{2}+\frac{r_{21}}{r_{12}+r_{21}} D_{1}\right) \approx p^{2} D_{e q}$. Therefore, Eq. (22) approximates to

$$
P\left(x, t \mid x_{0}, 0\right)=\frac{e^{-\frac{\left(x-x_{0}\right)^{2}}{4\left[D_{e q} t+D_{A}\left(t+\tau_{A}\left(e^{-\frac{t}{\tau_{A}}}-1\right)\right)\right]}}}{\sqrt{4 \pi\left[D_{e q} t+D_{A}\left(t+\tau_{A}\left(e^{-\frac{t}{\tau_{A}}}-1\right)\right)\right]}}
$$




\section{Diffusing diffusivity model}

In the short-time limit, i.e., for $\beta t \ll 1$, and for small and intermediate displacements (i.e., $\left.D_{e q} p^{2} / \omega \gg 1\right)$, the above can be approximated as

$$
\begin{aligned}
& P\left(x, t \mid x_{0}=0,0\right) \approx \frac{1}{2 \pi} \int_{-\infty}^{\infty} d p \frac{e^{-i p x}}{1+D_{e q} p^{2} t} e^{-D_{A} p^{2}\left(t+\tau_{A}\left(e^{-\frac{t}{\tau_{A}}}-1\right)\right)} \\
& \approx \frac{1}{2 \sqrt{D_{e q} t}} \sqrt{\frac{1}{4 \pi\left[D_{A}\left(t+\tau_{A}\left(e^{-\frac{t}{\tau_{A}}}-1\right)\right)\right]}} \\
& \int_{-\infty}^{\infty} d x^{\prime} e^{-\frac{\left|x-x^{\prime}\right|}{\sqrt{D_{e q} t}}} e^{-\frac{x^{\prime 2}}{4\left[D_{A}\left(t+\tau_{A}\left(e^{-\frac{t}{\tau_{A}}}-1\right)\right)\right]}} \\
& \approx \frac{e^{\frac{D_{A}\left(t+\tau_{A}\left(e^{-\frac{t}{\tau_{A}}}-1\right)\right)}{D_{e q t}}}}{2 \sqrt{D_{e q} t}} \cosh \left(\frac{x}{\sqrt{D_{e q} t}}\right) \\
& -\frac{e^{\frac{D_{A}\left(t+\tau_{A}\left(e^{-\frac{t}{\tau_{A}}}-1\right)\right)}{D_{e q t}}}}{2 \sqrt{D_{e q} t}} \operatorname{erf}\left(\frac{x}{\sqrt{\frac{4\left[D_{A}\left(t+\tau_{A}\left(e^{-\frac{t}{\tau_{A}}}-1\right)\right)\right]}{\sqrt{D_{e q} t}}}}\right) \sinh \left(\frac{x}{\sqrt{D}}\right) .
\end{aligned}
$$

In the long $t$ limit, i.e., for $\beta t \gg 1$, the PDF [Eq. (25)] can be approximated as

$$
\begin{aligned}
& P\left(x, t \mid x_{0}=0,0\right) \approx \frac{1}{2 \pi} \int_{-\infty}^{\infty} d p e^{-i p x} e^{-D_{e q} p^{2} t} e^{-D_{A} p^{2}\left(t+\tau_{A}\left(e^{-\frac{t}{\tau_{A}}}-1\right)\right)} \\
& \approx \sqrt{\frac{1}{4 \pi\left[D_{e q} t+D_{A}\left(t+\tau_{A}\left(e^{-\frac{t}{\tau_{A}}}-1\right)\right)\right]}} e^{-\frac{x^{2}}{4\left[D_{e q} t+D_{A}\left(t+\tau_{A}\left(e^{-\frac{t}{\tau_{A}}}-1\right)\right)\right]} .}
\end{aligned}
$$

[1] M. A. Thompson, J. M. Casolari, M. Badieirostami, P. O. Brown and W. Moerner, Proc. Natl. Acad. Sci. U.S.A., 2010, 107, 17864-17871.

[2] H. Shen, L. J. Tauzin, R. Baiyasi, W. Wang, N. Moringo, B. Shuang and C. F. Landes, Chem. Rev., 2017, 117, 7331-7376.

[3] S. Ramaswamy, Annu. Rev. Condens. Matter Phys., 2010, 1, 323-345.

[4] É. Fodor and M. C. Marchetti, Physica A, 2018, 504, 106-120.

[5] C. Maggi, M. Paoluzzi, N. Pellicciotta, A. Lepore, L. Angelani and R. Di Leonardo, Phys. Rev. Lett., 2014, 113, 238303. 
[6] L. L. Bonilla, Phys. Rev. E, 2019, 100, 022601.

[7] D. Martin, J. O’Byrne, M. E. Cates, É. Fodor, C. Nardini, J. Tailleur and F. van Wijland, Phys. Rev. E, 2021, 103, 032607.

[8] S. Joo, X. Durang, O.-c. Lee and J.-H. Jeon, Soft Matter, 2020, 16, 9188-9201.

[9] J. Um, T. Song and J.-H. Jeon, Front. Phys., 2019, 7, 143.

[10] S. Chaki and R. Chakrabarti, Physica A, 2018, 511, 302-315.

[11] K. Goswami, Phys. Rev. E, 2019, 99, 012112.

[12] K. Goswami, Physica A, 2019, 525, 223-233.

[13] S. Chaki and R. Chakrabarti, Physica A, 2019, 530, 121574.

[14] K. Goswami, Physica A, 2021, 566, 125609.

[15] B. Wang, S. M. Anthony, S. C. Bae and S. Granick, Proc. Natl. Acad. Sci. U.S.A., 2009, 106, 1516015164.

[16] J. Guan, B. Wang and S. Granick, ACS Nano, 2014, 8, 3331-3336.

[17] G. Kwon, B. J. Sung and A. Yethiraj, J. Phys. Chem. B, 2014, 118, 8128-8134.

[18] P. Kumar, L. Theeyancheri, S. Chaki and R. Chakrabarti, Soft Matter, 2019, 15, 8992-9002.

[19] I. Chakraborty, G. Rahamim, R. Avinery, Y. Roichman and R. Beck, Nano Lett., 2019, 19, 6524-6534.

[20] M. J. Skaug, J. Mabry and D. K. Schwartz, Phys. Rev. Lett., 2013, 110, 256101.

[21] I. Chakraborty and Y. Roichman, Phys. Rev. Research, 2020, 2, 022020.

[22] C. Xue, X. Zheng, K. Chen, Y. Tian and G. Hu, J. Phys. Chem. Lett., 2016, 7, 514-519.

[23] N. Samanta and R. Chakrabarti, Soft Matter, 2016, 12, 8554-8563.

[24] A. DÃez FernÃjndez, P. Charchar, A. G. Cherstvy, R. Metzler and M. W. Finnis, Phys. Chem. Chem. Phys., 2020, 22, 27955-27965.

[25] B. Wang, J. Kuo, S. C. Bae and S. Granick, Nat. Mater., 2012, 11, 481-485.

[26] M. V. Chubynsky and G. W. Slater, Phys. Rev. Lett., 2014, 113, 098302.

[27] E. Barkai and S. Burov, Phys. Rev. Lett., 2020, 124, 060603.

[28] A. M. Obukhov, J. Geophys. Res. (1896-1977), 67, 3011-3014.

[29] A. M. Oboukhov, J. Fluid Mech., 1962, 13, 77-81.

[30] A. N. Kolmogorov, J. Fluid Mech., 1962, 13, 82-85.

[31] A. Naert, L. Puech, B. Chabaud, J. Peinke, B. Castaing and B. Hebral, J. phys., II France, 1994, 4, 215-224.

[32] C. Beck and E. G. Cohen, Physica A, 2003, 322, 267-275. 
[33] R. Zwanzig, Acc. Chem. Res., 1990, 23, 148-152.

[34] A. Debnath, R. Chakrabarti and K. L. Sebastian, J. Chem. Phys., 2006, 124, 204111.

[35] N. Tyagi and B. J. Cherayil, J. Phys. Chem. B, 2017, 121, 7204-7209.

[36] R. Jain and K. L. Sebastian, J. Phys. Chem. B, 2016, 120, 3988-3992.

[37] R. Jain and K. L. Sebastian, J. Phys. Chem. B, 2016, 120, 9215-9222.

[38] R. Jain and K. L. Sebastian, Phys. Rev. E, 2017, 95, 032135.

[39] A. V. Chechkin, F. Seno, R. Metzler and I. M. Sokolov, Phys. Rev. X, 2017, 7, 021002.

[40] R. Metzler, Biophys. J., 2017, 112, 413.

[41] V. Sposini, A. V. Chechkin, F. Seno, G. Pagnini and R. Metzler, New J. Phys., 2018, $20,043044$.

[42] L. Luo and M. Yi, Phys. Rev. E, 2018, 97, 042122.

[43] L. Luo and M. Yi, Phys. Rev. E, 2019, 100, 042136.

[44] E. B. Postnikov, A. Chechkin and I. M. Sokolov, New J. Phys., 2020, 22, 063046.

[45] Y. Lanoiselée, N. Moutal and D. S. Grebenkov, Nat. Commun., 2018, 9, 1-16.

[46] V. Sposini, D. S. Grebenkov, R. Metzler, G. Oshanin and F. Seno, New J. Phys., 2020, 22, 063056.

[47] J. Ślęzak and S. Burov, Sci. Rep., 2021, 11, 1-18.

[48] S. Burov and E. Barkai, Phys. Rev. Lett., 2007, 98, 250601.

[49] R. Yamamoto and A. Onuki, Phys. Rev. Lett., 1998, 81, 4915-4918.

[50] J. S. Leith, A. Tafvizi, F. Huang, W. E. Uspal, P. S. Doyle, A. R. Fersht, L. A. Mirny and A. M. van Oijen, Proc. Natl. Acad. Sci. U.S.A., 2012, 109, 16552-16557.

[51] B. R. Parry, I. V. Surovtsev, M. T. Cabeen, C. S. O'Hern, E. R. Dufresne and C. Jacobs-Wagner, Cell, 2014, 156, 183-194.

[52] L. Cuculis, Z. Abil, H. Zhao and C. M. Schroeder, Nat. Commun., 2015, 6, 1-11.

[53] S. Mora and Y. Pomeau, Phys. Rev. E, 2018, 98, 040101.

[54] T. Miyaguchi, T. Akimoto and E. Yamamoto, Phys. Rev. E, 2016, 94, 012109.

[55] P. C. Bressloff, J. Phys. A: Math. Theor., 2017, 50, 133001.

[56] G. Mongillo, D. Hansel and C. van Vreeswijk, Phys. Rev. Lett., 2012, 108, 158101.

[57] A. Godec and R. Metzler, J. Phys. A: Math. Theor., 2017, 50, 084001.

[58] S. Paul, S. Ghosh and D. S. Ray, J. Stat. Mech.: Theory Exp., 2018, 2018, 033205.

[59] I. Santra, U. Basu and S. Sabhapandit, Phys. Rev. E, 2021, 104, L012601.

[60] E. Yamamoto, T. Akimoto, A. Mitsutake and R. Metzler, Phys. Rev. Lett., 2021, 126, 128101.

[61] S. Nampoothiri, E. Orlandini, F. Seno and F. Baldovin, Phys. Rev. E, 2021, 104, L062501. 
[62] F. Baldovin, E. Orlandini and F. Seno, Front. Phys., 2019, 7, 124.

[63] T. J. Lampo, S. Stylianidou, M. P. Backlund, P. A. Wiggins and A. J. Spakowitz, Biophys. J., 2017, $112,532-542$.

[64] A. G. Cherstvy, S. Thapa, C. E. Wagner and R. Metzler, Soft Matter, 2019, 15, 2526-2551.

[65] W. Wang, F. Seno, I. M. Sokolov, A. V. Chechkin and R. Metzler, New J. Phys., 2020, 22, 083041.

[66] B. P. Bhowmik, I. Tah and S. Karmakar, Phys. Rev. E, 2018, 98, 022122.

[67] J. M. Miotto, S. Pigolotti, A. V. Chechkin and S. Roldán-Vargas, Phys. Rev. X, 2021, 11, 031002.

[68] O. Chepizhko, E. G. Altmann and F. Peruani, Phys. Rev. Lett., 2013, 110, 238101.

[69] M. Bley, P. I. Hurtado, J. Dzubiella and A. Moncho-JordÃ $i$, Soft Matter, 2022, 18, 397-411.

[70] C. O. Reichhardt and C. Reichhardt, New J. Phys., 2018, 20, 025002.

[71] R. Sahoo, L. Theeyancheri and R. Chakrabarti, Soft Matter, 2022, 18, 1310-1318.

[72] C. Bechinger, R. Di Leonardo, H. Löwen, C. Reichhardt, G. Volpe and G. Volpe, Rev. Mod. Phys., 2016, 88, 045006.

[73] J.-L. Thiffeault and J. Guo, arXiv preprint arXiv:2102.11758, 2021.

[74] S. M. J. Khadem, N. H. Siboni and S. H. L. Klapp, Phys. Rev. E, 2021, 104, 064615.

[75] S. Chaki and R. Chakrabarti, Soft Matter, 2020, 16, 7103-7115.

[76] D. A. Garcia, G. Fettweis, D. M. Presman, V. Paakinaho, C. Jarzynski, A. Upadhyaya and G. Hager, Nucleic Acids Research, 2021, 49, 6605-6620.

[77] K. Goswami and K. L. Sebastian, Phys. Rev. E, 2020, 102, 042103.

[78] H. Risken, The Fokker-Planck Equation, Springer, 1996, pp. 63-95.

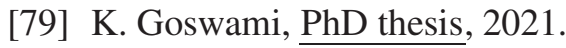

[80] K. Goswami and K. L. Sebastian, J. Stat. Mech.: Theory Exp., 2019, 2019, 083501.

[81] A. Pacheco-Pozo and I. M. Sokolov, Phys. Rev. Lett., 2021, 127, 120601.

[82] T. Uneyama, T. Miyaguchi and T. Akimoto, Phys. Rev. E, 2015, 92, 032140.

[83] N. Ahamad and P. Debnath, Physica A, 2020, 549, 124335.

[84] V. Balakrishnan, Pramana, 1993, 40, 259-265. 